\title{
ON A SIMPLE METHOD FOR THE APPROXIMATE DETERMINATION OF BORIC ACID.
}

By Cecil H. Cribb, B.Sc. (Lond.), F.I.C., and F. W. F. Arnaud, F.I.C.

(Read at the Meeting, March 7, 1906.)

THE process about to be described is not intended to replace any of the plans at present in vogue for the determination of boric acid, but has been found very useful as a check on the results obtained by other methods, especially in those cases where the quantity of the sample at the disposal of the analyst is very small.

The Thomson process, as employed for milk, besides being somewhat lengthy, has the further disadvantages of using up a large volume of the sample, and of not being diagnostic of boric acid. The results may be made to vary considerably by slight differences of procedure, and consequently a duplicate determination by the same or some other method is necessary. In many instances the bulk of the sample does not admit of this.

In the case of butter and cream, some plan of rapidly sorting out those samples having more than 0.5 per cent. and 0.25 per cent. respectively of boric acid from those having less is also a desideratum, which even the modification of the Thomson process, proposed by Richmond, does not quite supply.

The method now being brought forward has no claim to superior accuracy, and does not embody any new principle, being based on the well-known colour reaction with turmeric. Several processes have already been published which make use of this, but, as far as our knowledge goes, not one of them has met with general acceptance. That of Cassal and Gerrans (British Food Journal, 1902, p. 210) was the first in which any attempt was made to increase the delicacy of the colour reaction, and oxalic acid was added to the turmeric with this object. We found the process as a whole much too lengthy for general use, but the addition of the oxalic acid 
undoubtedly gave a greatly increased sensitiveness even to turmeric-paper, and the possibilities thereby opened up led us to make further experiments.

Another method based on the change in colour of a turmeric decoction produced by boric acid is that of Hebebrand (Zeit. f. Untersuch. Nahr. u. Genuss., 1902, 55-58), which consists in comparing the colour given by the sample under examination, after, of course, suitable preparation, with that produced under precisely similar circumstances, by known volumes of a standard solution of boric acid. We found, however, that besides wasting a good deal of alcohol and hydrochloric acid, the process was greatly lacking in delicacy.

As we had found that the addition of oxalic acid, as recommended by Cassal and Gerrans, added to the sensitiveness of turmeric-paper as well as to that of the decoction, we prepared papers with a number of other substances, and finally came to the conclusion that tartaric acid gave the best results, and that a paper prepared in the following manner was sufficiently delicate for the end we had in view : Take 2 parts by weight of turmeric and 2 of tartaric acid, and digest with 100 volumes of hot alcohol (about 80 per cent.) until all the tartaric acid has dissolved; then saturate fairly thick blotting-paper with the filtered solution, and hang up to dry, preferably in a dark place.

Paper made in this way turns, with strong solutions of boric acid, a bright rosepink colour, and the same colour is still just apparent when the dilution is as great as 0.0025 per cent., 2.5 parts per 100,000 .

To get the best results it is necessary to pay strict attention to a number of details. The turmeric-paper should preferably be fairly fresh, but we have found it keep its power without deteriorating for as long as a month. It is better preserved in the dark. The degree of acidity of the solution to be tested is of considerable importance. Tartaric turmeric-paper will give excellent results with boric acid alone (but not with borax), although ordinary turmeric hardly changes colour at all under these circumstances. We find, however, that the addition of a small proportion of hydrochloric acid considerably increases the sensitiveness, and in practice we have adopted 2 per cent. as the standard strength. If more is used the paper chars when dried, even at a moderate temperature; and with as little as 2 per cent. the drying operation should be conducted slowly-i.e., at a temperature under $60^{\circ} \mathrm{C}$.- and is best done in the dark.

Having prepared a sensitive paper, it only remains to apply it in such a way as to get quantitative results.

A number of possible plans will at once suggest themselves, and we shall content ourselves with giving merely as illustrations the actual method of working which we have adopted (1) as a check on other and more accurate methods in the case of milk, and (2) for controlling the proportion of boric acid in butter and cream.

1. Milk.-As the process is mainly intended for use in those cases where the quantity of the sample does not admit of a duplicate determination by the Thomson or other method, we use only a small volume, generally 5 c.c., but even less can be made to do. This quantity is mixed with 1 c.c. of normal alkali, and the whole is evaporated to dryness and incinerated. One c.c. of normal acid is added to the ash, which need not be absolutely white, and the volume is made up to $5,10,20$, or 30 or 
more c.c., according to the proportion indicated by the previous estimation, with 2 per cent. hydrochloric acid. A small strip of paper, 2 inches by $\frac{1}{2}$ inch, is then dipped into the liquid and dried in the dark at a gentle heat. If the paper then exhibits the rose-pink colour, the solution is diluted still further, and each dilution is tested with the turmeric-paper until a stage is reached when it fails to give the characteristic reaction at all. If the degree of sensitiveness is known, it is obvious that a simple calculation will be-sufficient to show whether the dilution is what it should be for the indicated percentage of boric acid.

To ascertain the degree of sensitiveness which need only be done at intervals, as the paper changes but slowly, a similar quantity of boric-acid free milk is treated precisely as above, after the addition of a known volume of a 1 per cent. solution of boric acid, and the ash of this is examined by means of the turmeric-paper precisely as already described, and the degree of dilution noted.

To give an idea of the dilution possible, we may instance a recent case of milk which was found to contain, by Thomson's process, $0 \cdot 119$ per cent. of boric acid. Five c.c. of this, when treated in the above manner, bore the addition of 95 c.c. of 2 per cent. hydrochloric acid, and still just showed the colour with the turmericpaper.

2. In the case of butter and cream, the process is conducted on precisely the same lines. The amount of dilution of the ash of 1 gram of butter containing 0.5 per cent. of boric acid, or cream containing 0.25 per cent., using paper of average delicacy, is about 1,000 times in the case of butter and 500 times in the case of cream. If, therefore, the samples under examination bear a greater dilution than this, they must obviously contain more than 0.5 or 0.25 per cent. of boric acid respectively. Of course, it is in all cases necessary to ascertain the delicacy of the paper by testing it against a standard in the manner already described.

\section{Discussion.}

The President said that he did not quite understand the authors' remark that the Thomson process was not diagnostic of boric acid.

Mr. CRIBB said that, although, of course, presumptively the estimation was one of boric acid, it was, strictly speaking, only an estimation of acidity, and slight differences in procedure were sometimes followed by extraordinary differences in the results. It would not do to take the results given by the Thomson process as boric acid without making use of the turmeric reaction, or some similar qualitative test, to see that boric acid was actually there.

The President thought that, provided that boric acid was ascertained really to be present, the figure given by the Thomson process might safely be taken as an accurate representation of the quantity.

Mr. CRIBB said that sometimes there were considerable differences between the results obtained by different operators.

The President said that the figure obtained depended upon the exact point at which the solution ceased to be acid to methyl orange. This was somewhat difficult to determine. He should like to ask whether the increase in sensitiveness which accompanied the use of tartaric acid might not be also obtained by the addition of 
more hydrochloric acid, the process being in other respects worked in the ordinary way. He had noticed that, if insufficient acid were added, the reaction was not obtained. He could confirm what the authors had said as to the non-keeping properties both of turmeric-paper and of the alcoholic solution.

Mr. CRIBB said that, when more hydrochloric acid was used instead of tartaric acid, it was impossible to dry the paper in a reasonable time without charring it. The colour obtained with turmeric-paper when tartaric acid was present was, moreover, quite different from that given by paper prepared in the ordinary way.

Mr. P. A. E. RichaRds said that he had found the process valuable for sorting purposes, and very sensitive. As little as 5 grains of boric acid per gallon was easily recognised by it when working on 5 c.c., and he thought that with care even smaller quantities might be detected. It was important, however, to keep the hydrochloric acid at about the strength mentioned-namely, 2 per cent.

Dr. Rideal said that Mr. Cribb had been good enough to instruct him in this method a year or so ago, and he had used it with very satisfactory results.

Mr. CRIBB said that the colouring matter produced by the action of boric acid on turmeric seemed to be a more or less definite compound, though he did not think it had ever been fully investigated. They found that when an acid solution of boric acid (as dilute as 0.01 per cent.) was distilled, boric acid could be readily detected in the distillate, but the addition of turmeric decoction to the solution caused the boric acid to be partially held back on distillation.

Mr. F. J. LLOYD thought that it would save much time and would conduce to greater accuracy if the operation of burning with alkali could be dispensed with, the test being simply made by adding hydrochloric acid, filtering, and testing the filtrate.

The President remarked that it was quite easy to detect even as little as 1 or 2 grains of boric acid per gallon in milk without burning, the turmeric being simply applied directly to the milk after acidification.

Mr. CrIBB said that they had tried what Mr. Lloyd suggested. For qualitative purposes the turmeric test could, of course, be employed on the milk direct without further treatment, but to use the reaction quantitatively it was necessary to remove the fat. This meant dilution, precipitation of the proteids, and filtration, and the process at once became too complicated and troublesome.

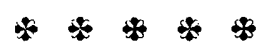

